\title{
PROPUESTA DE MODELO DE INTERVENCIÓN PSICOSOCIAL EN EL ADULTO MAYOR DESDE LOS ACERCAMIENTOS DE UN SEMILLERO DE INVESTIGACIÓN
}

\author{
Ruby Maritza Gerena $\mathrm{U}^{28}$ \\ Claudia Gracia, Yindeysi Espejo, Jacqueline Cano y Andrés Sánchez. ${ }^{29}$ \\ "Todos deseamos llegar a viejos y Todos negamos que hemos llegado" \\ Francisco de Quevedo y Villegas (Escritor Español)
}

\section{RESUMEN}

El presente artículo da a conocer una propuesta de atención psicosocial para aplicación en el adulto mayor, que busca mejorar la calidad de vida y promover la construcción de la comunidad como sujeto de derecho conocedor y participante de las políticas públicas. Esta surge en el contexto del semillero de investigación de Sofistas de la edad de Plata, desde la consejería y el programa de psicología de la UNAD direccionado a la reflexión y el trabajo del adulto mayor y desde las acciones previas del semillero; la revisión de estado del arte y la publicación previa de un libro sobre el tema, se continua con la formulación de una propuesta de intervención partiendo de un planteamiento evolucionista y constructivo de la vejez, resaltando la importancia del proyecto de vida y el mantenimiento de redes sociales como formas para la consecución de una mejor "calidad de vida".

Finalmente, se evidencia el planteamiento concreto de un modelo de intervención psicosocial desde la promoción y la prevención como estrategias que llevan a la inclusión social del adulto mayor.

Palabras clave: adulto mayor, envejecimiento, prevención, promoción, proyecto de vida, calidad de vida.

\begin{abstract}
This article reveals a proposal for implementation in psychosocial care for the elderly, and young adults, which seeks to improve the quality of life and promotes community building as subjects of law experts and participants in public policy. This arises in the context of research seed Sophists Silver age, from counseling and psychology program UNAD directed to the reflection and work of the elderly. From the previous actions of the seedbed as the state of the art review and publication of a book on the subject, continuing with the development of a proposed intervention based on an evolutionary

28 Psicóloga Universidad Nacional de Colombia, Especialización en Pedagogía UNAD, Investigadora principal semi1lero Sofistas de la edad de plata. Consejera académica de la UNAD-ZCBC. E-mail:ruby.gerena@unad.edu.co

29 Estudiantes de psicología, auxiliares de investigación.
\end{abstract}


and constructive approach of old age by stressing the importance of the draft life, maintaining social networks as ways to achieve a better quality of life".

Finally, the approach concrete evidence of a psychosocial intervention model from promotion and prevention as strategies that lead to social inclusion the elderly.

Key words: elderly, aging, prevention, advocacy, life planning, quality of life.

Recibido: 30 de marzo de 2009

Aceptado: 1 de agosto de 2009

\section{INTRODUCCIÓN}

La población adulta mayor de Colombia está creciendo aceleradamente. El Departamento Administrativo Nacional de Estadística DANE, señala, en el censo realizado en el año 2005, que el $6.3 \%$ de la población total de Colombia es mayor de 65 años. Para el 2050 se presupuesta que el total de la población esté cercano a los 72 millones de personas con una esperanza de vida superior a los 79 años, con más del 20\% por encima de los 60 años; será el segundo grupo poblacional de mayor crecimiento luego de la franja etaria comprendida entre los $40 \mathrm{y}$ los 60 años. (Proyecciones de Población Dane, 2005).

Los imaginarios sociales que se tienen frente a esta etapa de la vida como la disminución de las capacidades físicas y mentales (reales o no), su exclusión del campo laboral y por ende del social, los pone en una condición de desventaja y vulnerabilidad, aumenta la dependencia de sus familias, la invisibilidad social, el maltrato y los convierte en actores sociales propensos a ser excluidos o a excluirse. Con la situación planteada por el DANE es importante la preparación de la sociedad para asumir las transformaciones sociales y económicas que se generaran debido a estos cambios poblacionales.

La presente investigación parte del marco teórico realizado desde el semillero de investigación en Adulto Mayor, complementado con la indagación sobre imaginarios sociales y políticas públicas concernientes a las personas de la tercera edad, y desde la reflexión realizada acerca de nuestra vejez, lo que nos permite tener una relación cercana con el tema de investigación; la finalidad del trabajo es concluir en una propuesta de modelo de intervención desde la Psicología Social Comunitaria que permita a los adultos mayores constituirse en actores participantes de su realidad, como una forma de actuar frente al estado de vulnerabilidad y de exclusión en el cual se encuentran insertos.

A partir de estas investigaciones y revisión bibliográfica se planteó la posibilidad de diseñar, desde la Psicología Comunitaria, un proyecto de intervención que permitiera que los adultos mayores se reconocieran y se les identifique como sujetos activos dentro de la sociedad, con derechos y con la posibilidad de hacerse partícipes en el proceso de la construcción de la calidad de vida que desean tener, a través de un proyecto de vida. 
La prevención puede ser trabajada desde cualquier edad para permitir la planeación de la calidad de vida que se desea tener y dejar de lado la improvisación, la no planificación y como lo plantea Martin Baró en su definición de ambiente fatalista latinoamericano, aceptando con resignación el destino que nos tocó vivir.

El proceso de envejecimiento, por ser una construcción social, se encuentra atravesado por los imaginarios sociales que de ella se han tejido; es el momento en el cual los adultos mayores se han convertido en una carga para la familia y especialmente para sus cuidadores, ya que al no ser activos económicamente, no aportan en dinero al mantenimiento de la familia, aunque lo hagan con trabajo, es el caso de las abuelas que cuidan a sus nietos, sin remuneración económica. Estas personas no tienen metas propias, sino actos de servicio a sus hijos o su familia, por que la idea del fin y la incertidumbre que esto trae, no permite la realización de planes a futuro y los convierte en conformistas según lo encontrado en el estudio La sexualidad en la vejez: vivencia del imaginario en el 2002, realizado por la Universidad Javeriana en adulos mayores de Soacha y Sibaté.

Las representaciones sociales generan creencias acerca de la vejez; identifican a las personas que llegan a esa etapa como dependientes, frágiles, que prefieren la soledad. Las políticas públicas, manifiestas o no, acerca de la productividad laboral permiten la exclusión social de estas personas, las confina a ser una carga económica para sus familias y el estado, aumentan los problemas económicos y con esto los de violencia intrafamiliar. De esta forma, la pregunta que rige esta propuesta es: ¿Qué estrategias de intervención psicosocial pueden fortalecer la inclusión del adulto mayor, como actor participante y cambiante de su realidad social, que le permitan constituirse en sujeto social de derecho?

\section{CONTEXTO}

El aumento de la esperanza de vida, la disminución de la mortalidad, el control de las enfermedades infecciosas y parasitarias, el descenso de las tasas de la fecundidad, la migración de los jóvenes, son factores que permiten afirmar que la población del adulto mayor va en aumento y se convirte en un problema social, económico, político y cultural debido a las precarias políticas que existen, aunado a las representaciones sociales que se tienen del adulto mayor en nuestra sociedad; esta problemática no solo se encuentra en nuestro país, sino que a nivel mundial se nota una preocupación creciente acerca de este tema, reflejada en múltiples estudios que se llevan a cabo desde diferentes líneas del conocimiento, como en la medicina, la cual ya tiene una especialización en los problemas médicos propios del adulto mayor, la geriatría.

Al realizar la indagación acerca de las investigaciones hechas en Colombia acerca de la tercera edad se encontró que 19 universidades del país realizaron o se encuentran realizando investigaciones que se enmarcan en tesis, líneas de investigación inscritas en Conciencias, desde carreras sociales, médicas, económicas reflejando la necesidad de investigaciones acerca de los procesos de envejecimiento. 
Desde nuestro campo de estudio, la psicología, se han realizado investigaciones sobre diferentes temas acerca de problemáticas que aparecen en la tercera edad por nombrar un ejemplo se puede mencionar la experiencia de la Pontificia Universidad Javeriana a través del Instituto del envejecimiento, que se especializa en tratamientos para la tercera edad en un trabajo interdisciplinario aportando soluciones a múltiples problemas desde campos como la medicina, la psicología, los problemas de adecuación de vivienda, etc.

Los trabajos consultados enmarcan los problemas de la vejez, desde el paradigma de la enfermedad de todo tipo, dolencias, achaques, problemas psiquiátricos, etc., pero no hay muchos trabajos en el país desde la perspectiva de la Psicología Social Comunitaria, que permitan al adulto mayor, constituirse en un ser de derecho, al cual se debe incluir como sujeto activo de la sociedad. Por esta razón, se plantea la necesidad de realizar investigaciones que permitan deconstruir los imaginarios que generan creencias acerca de la vejez y la convierten en un estado frágil del desarrollo humano, lo que posibilita la exclusión social y la pérdida de derechos y posibilidades.

El tema reviste tal importancia que países considerados viejos en el continente, como Argentina y Uruguay y los desarrollados económicamente, como los europeos debido a las grandes tasas de población adulta que tiene en el momento, han trazado caminos para la consecución de "calidad de vida" en las personas de la tercera edad. Entre las ponencias expuestas en el VIII Congreso de Gerontología y Geriatría llevado a cabo del 16 al 19 de Abril en Cartagena, sobresalen "Políticas en Latinoamérica luego de la Asamblea del envejecimiento" y Sistemas de evaluación de los programas en comunidad, se plantea el desarrollo de programas desde la prevención para el mejoramiento de la calidad de vida.

El país y la sociedad se encuentran en mora de encontrar soluciones a los problemas económicos que vienen aunados a las políticas manejadas en materia laboral en este momento. El considerar a una persona vieja a los 40 años y sacarla del sistema laboral y económico nos conducirá a aumentar la pobreza y la miseria existentes. El estado no está preparado para garantizar a este gran número de personas un adecuado sistema de seguridad social que les permita vivir en condiciones óptimas, aportando desde su experiencia al desarrollo del país.

El proceso de construcción de nuevas creencias que permitan el empoderamiento como personas de derecho, debe partir de una autorreflexión acerca del proceso de envejecimiento, no mirándolo como la culminación de la vida, lo cual produce incertidumbre hacia el futuro (Hombrados, 2002), sino como una oportunidad de aprendizaje que se constituye en un proceso continuo y que no es marcado por tiempos cronológicos. El proceso social de estos cambios debe partir de un modelo de intervención diseñado desde la Psicología Social Comunitaria que plantee campos de reflexión de los actores y propuestas de solución que permitan trazar estrategias a largo plazo, para una inclusión de las personas de la tercera edad, realizando un trabajo de prevención en el adulto mayor de hoy y el de mañana. 
Este tema no debe solamente tratarse en el ahora, sino debe repensarse como una oportunidad de preparar a los jóvenes de hoy para que enfrenten su propio envejecimiento no como algo ajeno, sino una etapa de la carrera que todos debemos vivir.

\section{Evolución del proceso de envejecimiento}

Está demostrado que no todo el organismo envejece al mismo ritmo. Cada sistema orgánico tiene el suyo propio, a menudo influido por factores genéticos. Dentro del sistema nervioso central, los procesos degenerativos no son uniformes (Dichgans \& Schulz, 1999). Este factor debe tenerse en cuenta al hacer afirmaciones sobre el envejecimiento. La heterogeneidad, que es de regla en el comportamiento, se encuentra también en el substrato biológico de la conducta y la vivencia. Crucialmente, la vejez es etapa biográfica, señalada por ciertos atributos exteriores, de acuerdo con el reloj social que cada comunidad tiene. Internamente, esta etapa es marcada más por lo que se es que por lo que se hace.

Es frecuente encontrar personas afectadas por la opinión que de ellas tienen los otros, mantenida a lo largo de años, que contrasta con la opinión propia. Por esto se puede afirmar que el concepto de vejez es una construcción social que tiene sus bases en los imaginarios culturales que se elaboran sobre esta etapa, así como en épocas pasadas o en algunas culturas donde las personas viejas eran o son reverenciadas como portadoras de sabiduría, experiencia y de las tradiciones de las culturas. En este momento son consideradas inservibles debido a los modelos económicos que nos rigen, en los cuales el valor está dado por lo que se pueda aportar.

La vida del ser humano es definida desde diferentes puntos de vista: biológico, entendido por la posición y las expectativas respecto a la duración potencial de los años de vida y se acompaña de elementos que miden la capacidad funcional de los sistemas vitales. La posición presente y expectativa respecto a su duración potencial en años de vida, debe ser acompañada de elementos que midan la capacidad funcional de los sistemas vitales cuya situación limita o prolonga el ciclo. Es lógico entender que este tipo de edad es al que comúnmente le llamamos edad, con la única diferencia de que aquí no es cuántos llevas, sino cuántos te quedan, en términos de vida biológica.

Por otra parte, está el punto de vista de la Psicología que se relaciona con la capacidad de adaptación que manifiestan las personas ante los diferentes eventos de la vida, por lo que es algo similar a lo que se llama "madurez" en el lenguaje cotidiano, y de hecho esta capacidad se logra a través de los años con la experiencia que se va acumulando. La capacidad que el sujeto manifiesta para adaptarse a los distintos cambios estructurales se hace evidente hoy; los históricos por ejemplo, que una sociedad puede plantear al sujeto, puesto que en la vida de una persona se suscitan muchos cambios a nivel social, partiendo del hecho de que de una generación a otra se viven situaciones drásticamente diferentes y más en el presente que, con el agigantado avance de la ciencia y la tecnología, los estilos de vida cambian frecuentemente. 
Otro aspecto es el social que tiene que ver con los roles y hábitos sociales que el sujeto es capaz de asumir en relación con el término medio del contexto con los que desempeñarían otros miembros del grupo social y los que pudiera asumir en un momento dado. Este último papel se puede entender con mayor claridad a través de la sociología, en donde lo fundamental consiste en el tipo de roles que ocupan las personas en una estructura social. Por lo tanto, esta edad es definida por el rol que un sujeto ocupa en la sociedad, dentro del cual se involucra en las actividades del sujeto y su poder correspondiente.

Las Representaciones Sociales del adulto mayor también pueden ser evidenciadas en las teorías que se manejan sobre la vejez:

Teoría de la desvinculación o Teoría del desapego, expuesta por Cumming y Henry (1961) (Sentilecto Psique. 2007). A medida que la persona envejece se preocupa más por ella y pierde interés en el mundo que los rodea, llevándolos a aislarse voluntariamente y a creer que de esta manera no obstaculizarán el desarrollo de las generaciones más jóvenes, por ser un proceso universal, que se presenta en cualquier cultura y a través de los tiempos.

La Teoría de la Actividad, (Belando) planteada inicialmente por Havighurst, (también se habla del alemán Tartler (1961) y de Atchley (1977) como representante de esta teoría, a la vez que se ha observado en diferentes estudios como los de Maddox $(1963,1968))$, plantea que los viejos deben permanecer activos, en tanto ellos tengan los recursos para hacerlo. Por otra parte, si esta persona presenta cierto grado de dificultad, deben diseñarse sustitutos adecuados. Maddox cree que un factor importante es la personalidad previa pues es pieza clave para afrontar esta etapa. El autor estudió personas adultas mayores que hicieron descubrimientos, escribieron libros, fueron aristas o políticos conocidos. Debe quedar claro que al plantearse esta teoría se están reafirmando los imaginarios sociales sobre la calidad de vida y se está subrayando que los adultos mayores vulnerables no entrarían dentro de este planteamiento.

Desde la antropología San Román (1989) habla de "imagen idílica del anciano en el seno familiar de épocas pretéritas". Exactamente se dirige a la marginación diciendo que: "el anciano empieza a estar marginado cuando se le suplanta su rol social y esto ocurre ahora y ha ocurrido siempre”.(Sentilecto et al., 2007)

Rocio Fernández Ballesteros (1992) expresa que las percepciones y conceptualizaciones negativas del envejecimiento son infundadas, sumado a que el saber popular está lleno de dichos e imágenes que comparan a la vejez con déficit y deterioros físicos, psicológicos y sociales. Y cree que va a ser muy difícil conseguir una vejez saludable si los mismos viejos, la población en general y los profesionales de la medicina persisten en la articulación de estos preconceptos.

La doctora Fernández puntualiza que el ser humano no se termina psicológica, ni física, ni biológicamente, es decir, no hay deterioro. Ella argumenta que el desarrollo humano desde una perspectiva psicológica, dura mientras sigue produciendo las transacciones entre el organismo biológico y el contexto sociocultural, dentro de los cuales hay pérdidas y ganancias a lo largo del ciclo de la vida. 


\section{Propuesta modelo de intervención}

Las revisiones bibliográficas efectuadas en los temas de "los imaginarios de la vejez y en la vejez" sumado a las "Políticas públicas", la calidad de vida, además de una reflexión con base a una discusión personal y grupal acerca de lo que significa ser viejo, permitió que surgiera la identificación del objeto del trabajo que es la consecución de "calidad de vida" del adulto mayor desde su propia iniciativa.

La siguiente es la propuesta del diseño de un Modelo de Intervención el cual se construye a partir de una revisión bibliográfica de "los imaginarios de la vejez y en la vejez" y "Políticas públicas" referentes a este sector de la población, además de una reflexión y discusión personal y grupal acerca de lo que significa ser "viejo".

Prevención para el adulto mayor: con el conocimiento de las representaciones sociales de esta población, acerca del tema, podemos trabajar en campos de prevención y previsión propuestas desde una política pública en educación en todas las etapas cronológicas de la vida, es decir, desde niños hasta adultos en la reflexión y concienciación del tema del adulto mayor, realizando programas de conocimiento y comprensión de los imaginarios existentes en esta etapa, resaltando la importancia del diseño de un proyecto de vida en la realización de una persona y partiendo de la premisa de que los jóvenes de hoy serán encargados del manejo de la situación futura y de su propio envejecimiento. La pregunta pertinente sería: ¿Están preparándose para ese reto?

Adulto mayor actual: dentro de este marco de población ha de considerarse un modelo de intervención, que a través de la educación en dos frentes: los cambios dados este proceso de la vida y el conocimiento de los derechos que afectan su calidad de vida y se encuentran representados en recreación, salud, prebendas en sitios públicos, le permitan un proceso de empoderamiento como sujetos de derecho y actores activos de la sociedad en la que se encuentran.

\section{Propuesta de trabajo de educación y prevención para los adultos jóvenes}

Desde el modelo socio comunitario, el cual plantea que los cambios sociales se pueden realizar desde la concienciación del individuo, se propone la realización de una propuesta de modelo de intervención que conciencie al joven de hoy que se es viejo desde el momento en que se nace, es por esto que esta propuesta se denominó "El adulto mayor del mañana" como un llamado de atención al hecho de que en el futuro de todos se encuentra la vejez. (Ver gráfica 1).

Con la educación se podría replantear el discurso "de que el que envejece es el "otro", a partir de políticas públicas de prevención en la preparación del adulto mayor del mañana y como una forma de disposición más objetiva por estar centrada en el individuo, para enfrentar la vejez. La propuesta plantea el trabajo desde la infancia de un "proyecto de vida" que le otorgue a la existencia, trascendencia y le quite la visión inmediatista del hoy y del ahora, 
bajo la premisa de que los actos hechos en el presente van a incidir en la "calidad de vida" que se va a tener en el mañana. Esta calidad tiene que ver con ejes como: la posibilidad de previsión, la autoría y el control de la vida

Gráfica 1. Propuesta de trabajo de educación y prevención para los adultos mayores del mañana

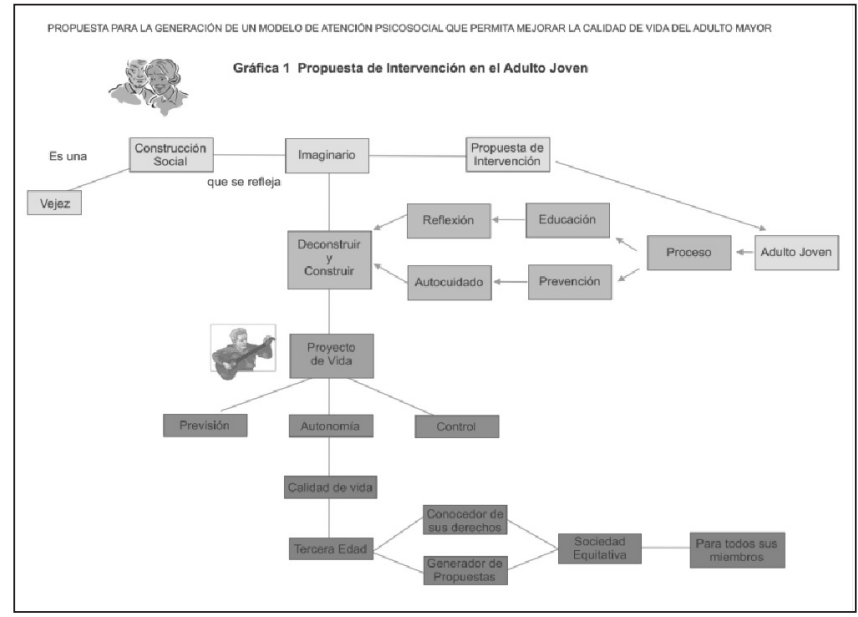

Gráfica 2. Propuesta de trabajo de educación y prevención para los adultos jóvenes

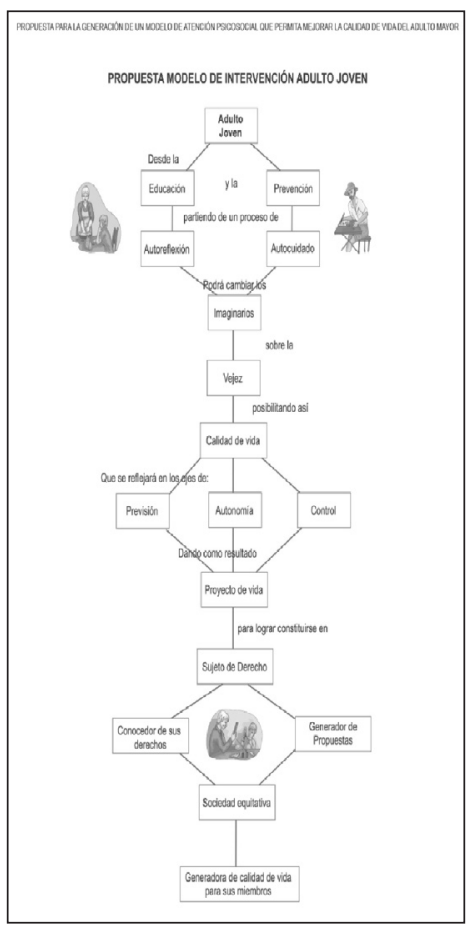




\section{Propuesta modelo de intervención en adulto mayor}

La siguiente propuesta de intervención se denomina "Viviendo mi vejez desde hoy" y es un reconocimiento a que el proceso de envejecimiento es algo que afrontamos todos los días de la vida a partir del nacimiento y no está marcado por una edad cronológica socialmente impuesta. Este modelo brinda otras oportunidades de buscar alternativas enriquecedoras para la existencia.

El Objetivo buscado es lograr el empoderamiento como sujetos de derecho (conocimiento de las políticas públicas generadas en su favor y formas de gestión con que se pueden lograr otras, para su inclusión como sujetos activos en la sociedad) de este sector de la población, a través de la educación en el proceso que están viviendo.

El modelo de intervención propuesto tendrá en cuenta el concepto de "calidad de vida", importancia del diseño de un proyecto de vida, fortificación de las redes sociales y la construcción de un proyecto de producción.

Desde la Educación trabajada a partir de talleres que informen y den una clara idea de los cambios presentados en esta etapa, el adulto mayor los identificaría en su vida asumiéndolos como un proceso natural de la existencia de todos y no la "enfermedad" que le tocó sufrir solo a él. La familia como red primaria de apoyo sería partícipe de este proceso de educación, enlazando la propuesta de intervención el adulto mayor del mañana. En esta etapa se busca lograr la Concienciación del adulto mayor y de su familia permitiendo una evaluación y autoevaluación de los conceptos que se tiene sobre la vejez con el propósito de modificar estos imaginarios que los sumen en actitudes de desesperanza frente al futuro.

Luego se propondrá la realización de un Proyecto de vida resaltando la significación que tiene en la consecución de objetivos a corto, mediano y largo plazo, teniendo como base el conocimiento y análisis de la realidad, del entorno y de los recursos. Se trabajaría en diferentes etapas que partirían desde la capacitación en diferentes ítems que proporcionen el conocimiento de las etapas necesarias para la creación y montaje del proyecto productivo. Luego vendría la Generación de recursos que implica el entrenamiento en gestión para la realización de propuestas para el sector empresarial. Posteriormente tendríamos la concientización política vista como participación desde decisiones e inspiradores de políticas públicas que afectan su calidad de vida. Con este aspecto se logra el hacerse sujetos de derecho incluidos y participes de la sociedad, procurando una democracia activa y participativa. Y finalmente, la Generación de redes de pares que permitiría el crecimiento personal, (según lo expuesto en el aparte de redes), a través de la amistad posibilitando la realización del proyecto de vida en el cual se inserta el proyecto productivo, como una forma para constituirse en actores válidos ante la comunidad con propuestas y estrategias propias en la solución de sus problemas (empoderamiento).

Para la continuidad de este trabajo se constituirán Redes Multiplicadoras que desarrollen acciones de promoción sobre el proceso llevado a cabo demostrando, con su ejercicio, la 
relevancia del concepto de futuro en la calidad de vida de los adultos jóvenes y adultos mayores especialmente, garantizando la continuación del proyecto.

Gráfica 3. Propuesta Modelo de intervención en adulto mayor

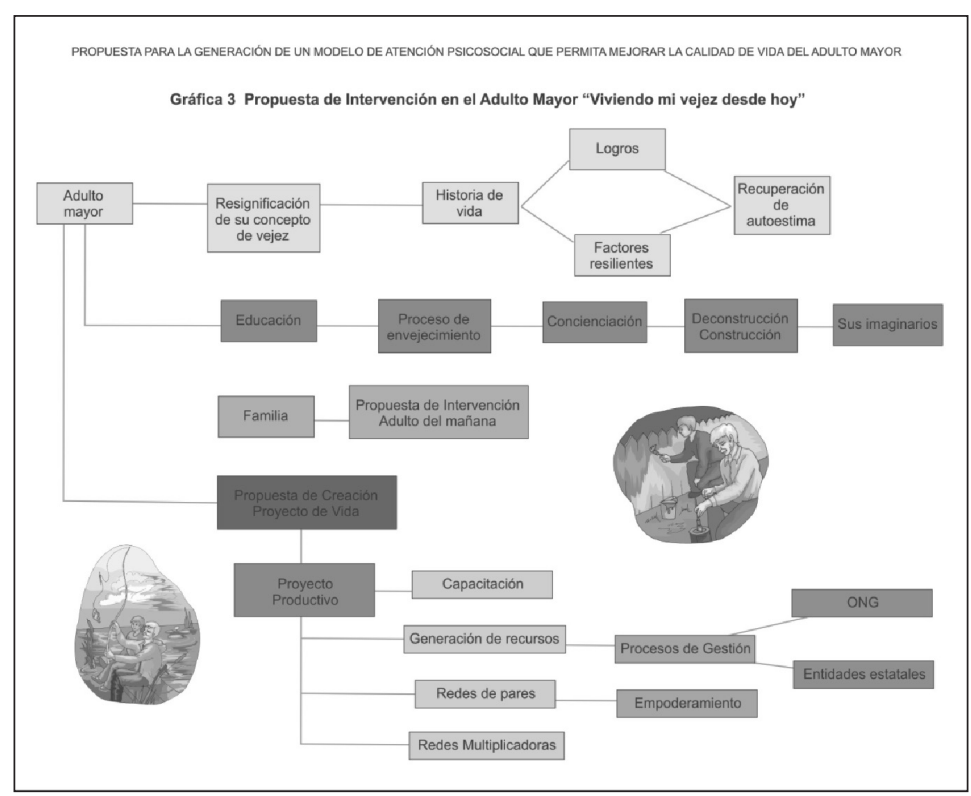

RESULTADOS DE LA INVESTIGACIÓN

A partir del estudio y seguimiento, realizado el ejercicio investigativo, formula como resultado la propuesta del diseño de un Modelo de Intervención el cual involucra dos fases:

a. Fase de Prevención: en donde se trabaja en un modelo de prevención y promoción dado desde una política pública en educación en todas las etapas cronológicas de la vida, es decir, desde niños hasta adultos en la reflexión y concientización del tema del adulto mayor, realizando programas de conocimiento y comprensión de los imaginarios existentes en esta etapa de la vida, resaltando la importancia del diseño de un proyecto de vida en la realización de una persona y partiendo de la premisa de que los jóvenes de hoy serán encargados del manejo de la situación futura y de su propio envejecimiento.

b. Adulto mayor actual: se considera un modelo de intervención que a través de la educación en dos frentes: los cambios dados en este proceso de la vida y el conocimiento de los derechos que afectan su calidad, le permitan un proceso de empoderamiento como sujetos de derecho.

Finalmente se muestran las propuestas como elementos catalizadores de una mejor realidad para el adulto mayor actual y los que llegarán allí. 


\section{CONCLUSIONES}

En la presente propuesta se logró conocer cómo desde los diferentes enfoques se intentan aproximaciones que pretenden el mejoramiento de la calidad de vida del adulto mayor. Se establecieron de esta manera dos modelos: el primero que abarca un trabajo preventivo con todos aquellos adultos de la actualidad cuya intención es el trabajo de hábito y acciones saludables que permitan llegar a una vejez digna. El segundo modelo plantea un trabajo a nivel de intervención desde la política pública y el mejoramiento de situaciones actuales que permitan que el adulto mayor actual trabaje sobre categorías como proyecto de vida, red de apoyo familiar y el aprendizaje.

Las investigaciones desde las cuales se fundamenta esta propuesta demuestran que "Una vida socialmente activa durante la vejez, en comparación con la de aquellas personas que mantienen escasos contactos sociales, se encuentran asociados a una mayor satisfacción con la propia vida, asi como a una mayor capacidad funcional" (OMS 1989), De esta manera, se contextualiza un ejercicio en donde la actividad social y la participación, son elementos críticos a la hora del empoderamiento de este grupo etario.

El asilamiento y la percepción final frente a la existencia, permiten que el adulto mayor no reformule su proyecto de vida, sin embargo, el poder trabajar en este punto ha permitido que se organice, participe y logre un mejoramiento significativo en su calidad de vida, a través del fortalecimiento de redes, organizaciones productivas y participación en política.

Se puede apreciar que muchas de las personas de la tercera edad en el país no tienen una garantía para el desarrollo integral de su autonomía. Las políticas públicas que se generan en relación con estas personas, no perciben sus reales necesidades ni las involucran en la toma de decisiones como una forma de reconocimiento a sus derechos.

De tal forma, que el propósito de conocer sobre el envejecimiento o la llegada a la edad adulta mayor es de gran importancia, ya que el estudiar el desarrollo de esta permitirá que se planteen estrategias que propendan por mejores ambientes y condiciones para los seres humanos puesto que en definitiva todos nos veremos afectados con este tipo de acciones cuando lleguemos a viejos.

\section{REFERENCIAS BIBLIOGRÁFICAS}

ADULTO MAYOR http://209.85.207.104/search?qcache:o4JdJ9Lx3uYJ:www.contraloriagen.gov. co:8081/internet/central_doc/Archivos/255/Inf_social2004_cuarta_parteA.pdfadultomayor2Bcol ombia2BbogotC3A12Bpolitica2Bpublica\&hles\&ctclnk\&cd1\&glo

BONILLA, NELSY. (1997). Mas allá del dilema de los métodos. La investigación en ciencias sociales. Colombia, Editorial Norma. 
BRONFRENBRENER (1987), La Ecología del desarrollo humano. Madrid: Paidos.

CALIDAD DE VIDA y Praxis Urbana Julio Alguacil Garavito disponible en http://habitat. aq.upm.es/

CÁMARA. http://direccion.camara.gov.co/camara/site/artic/20070514pags/200705141005. html.http://prensa.camara.gov.co/camara/site/artic/20070730/asocfile/006_07_camara ponencia_1er_dte.doc

CEDEÑO, DIÓGENES. (2007). Red latinoamericana de Gerontología. Panamá.

CONSEJODEBOGOTÁ.http://concejodebogota.gov.co/prontus_cbogota/antialonehtml?page=http:// concejodebogotagov.co/prontus_cbogota/site/edic/base/port/inicio.html

DANE CENSO. http://www.dane.gov.co/. - Proyecciones de Población

ERIKSON, E. La adultez. Madrid. Fondo de Cultura Económica.

DICHGANS \& SCHULZ. (1999). Citado por Fernando Lolas Stepke en "La vejez como etapa vital: consideraciones bioeticas" http://www.gerontologia.uchile.cl/docs/lolas_4.htm.

DIPLOMADO DE GERONTOLOGÍAA DISTANCIA. Programa para el Adulto mayor.2004. URL htt://www.inta/geratriados/

FAMILIA htt:/www.monografias.com/trabajo/antrofamilia/antrofamilia.shetml

FERNÁNDEZ-BALLESTEROS ROCÍO. (1992) La Psicología De La Vejez.1992. Disponible En La Web::Www.Encuentros-multidisciplinares.org/Revistan ${ }^{\circ} 16 /$ Rocío20Fernandez20Ballesteros.pdf.

FRANCISCO, CARMONA, MORENO LIGIA. (1990) La Ancianidad, Bogotá III Foro Nacional de Docentes e Investigadores Universitarios Sobre Envejecimiento Y Vejez. Agosto de 2004 II Jornadas Nacionales "La Vejez, Abordaje Interdisciplinario

GASTON, LILIANA. La vejez como objeto de las representaciones sociales. Disponible en la Web:http://bibliotecavirtual.clacso.org.ar/ar/libros/argentina/germani/gastron.rtf

GARCÍA, JOSÉ LUIS. (2005).’La sexualidad y la afectividad en la vejez”. Disponible:http:// www.imsersomayores.csic.es/documentos/documentos/garcia-sexualidad-01. pdf.

G.N. FISCHER. (1990). Psicología Social.

GRAU, J (1998). Calidad de vida y salud: Problemas actuales en su investigación. II Jornada de Actualización en Psicología de la Salud. Valle del Cauca. 
GRACIA, FUSTER ENRIQUE; HERRERO JUAN. (2005) Redes sociales de apoyo y ajuste biopsicosocial en la vejez un análisis comparativo en los contextos comunitario y residencial Revista sobre igualdad y calidad de vida Vol $14 \cdot 1$ disponible en http://www. uv.es/ egracia/enriquegracia/docs/scanner/Ajuste20vejez.pdf

GURLAND,B Y KATZ, S. (2000): "Calidad de vida y trastornos mentales de los ancianos".

HERNÁNDEZ, A. (2001). Familia, ciclo vital y psicoterapia breve. Bogotá: El búho

HERNÁNDEZ, ZAMORA ZOILA EDITH. "Estudio exploratorio sobre el proyecto de vida en el adulto mayor, Disponible en http://redalyc.uaemex.mex/pdf

HOMBRADOS MENDIETA MARÍA ISABEL. (1977). Introducción a la Psicología Comunitaria. Ed. Aljibe.

INSTITUTO DE ENVEJECIMIENTO - Universidad Javeriana http://medjaveriana.edu.co/ envejecimiento. Instituto Mexicano de Sexología A.C. "La sexualidad en la vejez: vivencia del imaginario". 2002

Martin Baró S.I. (1998). “Hacia una psicología de la liberación” (1998). Madrid: Trotta.

MINISTERIO DE COMUNICACIONES Y GRUPO INTERDISCIPLINARIO CONSULTOR DEL CENTRO DE PSICOLOGÍA GERENTOLÓGICA CEPSIGER. (Junio de 2004). Bogotá, D.C., Colombia. Envejecimiento Comunicación y Política Bogota D.C. Colombia

MINISTERIO DE PROTECCIÓN SOCIAL. http://www.minproteccionsocial.gov.co/ vbecontent/library/documents/DocNewsNo31DocumentNo1375.PDF

MUÑOZ, J. (1997): "Prevención comunitaria de las discapacidades en las personas mayores" en Buela- Casal, G (ed): Psicología Preventiva. Madrid: Pirámide.

NATHAN ACKERMAN DIAGNÓSTICO Y TRATAMIENTO DE LAS RELACIONES FAMILIARES. (1982). Edic. Hormé, Edit Paidós, $7^{\text {a }}$ edic, p. 21.

NEUGARTEN, B.L. (1996). Los significados de la edad [The meanings of age]. Barcelona: Herder.

ORGANIZACIÓN PANAMERICANA DE LA SALUD, Oficina Regional de la Organización Mundial de la Salud: ProMover: Un Estilo de Vida para las Personas Adultas Mayores. Washington D.C. 2002

PAPALIA, DIANE E; WENDKOS, SALLY. (1998). Desarrollo Humano con Aportaciones

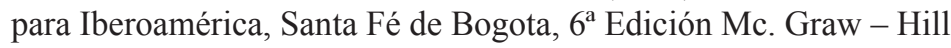


ROJAS, MALDONADO GUSTAVO. (Junio de 1986) Algunos aportes de la Psicología la gerontología: perspectivas de una línea de investigación. Conferencia Latinoamericana y del Caribe de Gerontología Perspectivas de una Línea de Investigación. Apartes de la Investigación y desarrollo U. del Norte Barranquilla.

SARTRE en Mishara, Riedel: 1986 El proceso de envejecimiento, Ed. Moratan. Madrid,.

SENTILECTO PSIQUE. (2007) .Vejez y Viejismo. Disponible En La Web :http://sentilecto. spaces.live.com/?_c11_BlogPart_BlogPart=blogview\&_c=BlogPart\&partqs $=$ cat3dVejez

SKOKNIC,V. (1999): "Efectos de la Preparación Psicológica para la Jubilación" en Luzoro,J (ed): Psicología de la Salud. Santiago: Bravo y Allende Editores.

STEVE BORGATTU. 14 de Febrero de 2003, Boston Collage www.analytictec.com.).

TRUJILLO, GARCÍA SERGIO. (2004). Formulación de un modelo teórico de la calidad de vida desde la psicología Revista Universidad Javeriana Psicológica No, V.

VERA MARTHA. (2007). Significado de la calidad de vida del adulto mayor para sí mismo y para su familia. Disponible en la Web: anales@unmsm.edu.pe.

VÁZQUEZ VIGO CARMEN. (1982). El miedo a los años. SM. Madrid.

ZAMIR SILVA AMIN. (2007) Informe de ponencia: Proyecto de Ley Estatutaria No. 006 